Received: 18 October 2016

Accepted: 22 February 2017

Published online: 27 March 2017
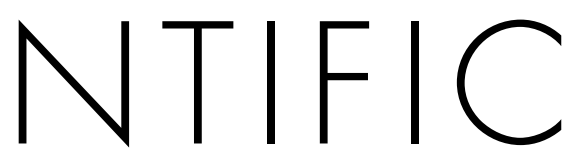

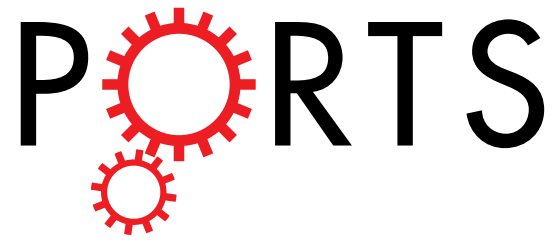

\title{
OPEN Combination of volume and perfusion parameters reveals different types of grey matter changes in schizophrenia
}

\author{
Lixue Xu ${ }^{1}$, Wen Qin $^{1}$, Chuanjun Zhuo ${ }^{2,3}$, Huaigui Liu ${ }^{1}$, Jiajia Zhu ${ }^{1}$ \& ChunshuiYu ${ }^{1}$
}

Diverse brain structural and functional changes have been reported in schizophrenia. Identifying different types of brain changes may help to understand the neural mechanisms and to develop reliable biomarkers in schizophrenia. We aimed to categorize different grey matter changes in schizophrenia based on grey matter volume (GMV) and cerebral blood flow (CBF). Structural and perfusion magnetic resonance imaging data were acquired in 100 schizophrenia patients and 95 healthy comparison subjects. Voxel-based GMV comparison was used to show structural changes, CBF analysis was used to demonstrate functional changes. We identified three types of grey matter changes in schizophrenia: structural and functional impairments in the anterior cingulate cortex and insular cortex, displaying reduction in both GMV and CBF; structural impairment with preserved function in the frontal and temporal cortices, demonstrating decreased GMV with normal CBF; pure functional abnormality in the anterior cingulate cortex and lateral prefrontal cortex and putamen, showing altered CBF with normal GMV. By combination of GMV and CBF, we identified three types of grey matter changes in schizophrenia. These findings may help to understand the complex manifestations and to develop reliable biomarkers in schizophrenia.

Schizophrenia is a neuropsychiatric disorder with complicated structural and functional changes, resulting in diverse clinical manifestations. The grey matter volume (GMV) is the most frequently used and reliable imaging index to characterize brain structural changes. GMV reduction has been considered as the most prominent structural changes in schizophrenia ${ }^{1}$. The reduced GMV has been found in multiple brain regions ${ }^{2-4}$ and in various schizophrenia related subpopulations $s^{5-7}$. The cerebral blood flow $(\mathrm{CBF})$ is a reliable imaging index to characterize brain functional changes. The CBF is closely coupled with glucose utilization and oxygen consumption, thus reflects the local neuronal activity ${ }^{8}$. Both increased and decreased regional CBF have been observed in schizophrenia $^{9,10}$.

Identifying different types of brain changes may improve our understanding of the neural mechanisms and facilitate the development of reliable biomarkers for schizophrenia. The combination of brain structural and functional changes may help to identify several types of meaningful regional brain alterations in schizophrenia. Recently, one study have applied structure and function parameters to distinguish schizophrenia patients from healthy comparison controls, and found that combination of structural and functional magnetic resonance imaging (MRI) data outperformed single MRI modality ${ }^{11}$, which implied that integration of structural and functional analysis could be more effective to describe the cerebral aberration of schizophrenia. However, no prior attempt has been done to combine volume and perfusion parameters to categorize brain change patterns in schizophrenia.

We assumed that there are at least three types of brain changes in schizophrenia. If brain regions exhibit both structural and functional impairments, these regions would probably be the primary causes for clinical symptoms. If brain regions show structural impairments with normal function or functional compensation, effective treatments may prevent these impairments from progressing into functional decompensation. And if structurally

${ }^{1}$ Department of Radiology and Tianjin Key Laboratory of Functional Imaging, Tianjin Medical University General Hospital, Tianjin, 300052, China. ${ }^{2}$ Tianjin Anding Hospital (Tianjin Mental Health Center), Tianjin, 300222, China. ${ }^{3}$ Tianjin Anning Hospital, Tianjin, 300300, China. Lixue Xu, Wen Qin and Chuanjun Zhuo contributed equally to this work. Correspondence and requests for materials should be addressed to C.Y. (email: chunshuiyu@tijmu.edu.cn) 
normal brain regions demonstrate functional abnormalities, pointed treatments may recover these abnormal functions.

In this study, the voxel-based GMV comparisons between patients and controls were used to identify structurally impaired brain regions in schizophrenia. In brain regions with GMV reduction, the voxel-based CBF comparisons were performed to further identify regions with and without CBF reduction. The similar CBF analysis was performed in brain regions without GMV reduction to detect pure functional abnormality in schizophrenia.

\section{Methods and Materials}

Participants. A total of 100 schizophrenia patients and 95 healthy comparison subjects were recruited for this study. Diagnoses for patients were confirmed using the Structured Clinical Interview for DSM-IV. The inclusion criteria included age (18-55 years), Chinese Han population, and right-handedness. The exclusion criteria included MRI contraindications, poor image quality, or a history of a systemic medical illness, CNS disorder or substance abuse. Additional exclusion criteria for the healthy comparison subjects included a history of any Axis I or II disorders, diagnosis of a psychotic disorder or a first-degree relative with a psychotic disorder. The clinical symptoms of psychosis were quantified with the Positive and Negative Syndrome Scale (PANSS) ${ }^{12}$. This study was approved by the Medical Research Ethics Committee at Tianjin Medical University General Hospital, and after complete description of the study, written informed consent was obtained from each participant. All methods were performed in accordance with the relevant guidelines and regulations.

Image data acquisition. MRI was performed using a 3.0-Tesla MR system (Discovery MR750, General Electric, Milwaukee, WI, USA). Tight but comfortable foam padding was used to minimize head motion, and earplugs were used to reduce scanner noise. Sagittal 3D T1-weighted images were acquired by a brain volume sequence with the following parameters: repetition time $(\mathrm{TR})=8.2 \mathrm{~ms}$; echo time $(\mathrm{TE})=3.2 \mathrm{~ms}$; inversion time $=450 \mathrm{~ms}$; flip angle $(\mathrm{FA})=12^{\circ}$; field of view $(\mathrm{FOV})=256 \mathrm{~mm} \times 256 \mathrm{~mm}$; matrix $=256 \times 256$; slice thickness $=1 \mathrm{~mm}$, no gap; and 188 sagittal slices. The resting-state perfusion imaging was performed using a three-dimensional pseudo-continuous arterial spin labeling imaging (pcASL) sequence with a 3D fast spin-echo acquisition and background suppression: $\mathrm{TR}=4886 \mathrm{~ms}$; $\mathrm{TE}=10.5 \mathrm{~ms}$; post-label delay $=2025 \mathrm{~ms}$; spiral in readout of eight arms with 512 sample points; $F A=111^{\circ}$; FOV $=240 \mathrm{~mm} \times 240 \mathrm{~mm}$; matrix $=128 \times 128$; slice thickness $=4 \mathrm{~mm}$, no gap; 40 axial slices; and number of excitation $=3$. During the ASL scans, all subjects were instructed to keep their eyes closed, to relax and move as little as possible, to think of nothing in particular, and to not fall asleep. After MRI scans, we ask subjects whether they have done as instructed.

GMV calculation. The GMV of each voxel was calculated using Statistical Parametric Mapping software (SPM8; http://www.fil.ion.ucl.ac.uk/spm/software/spm8/). The structural MR images were segmented into grey matter (GM), white matter and cerebrospinal fluid using the unified segmentation model. After an initial affine registration into Montreal Neurological Institute (MNI) space, GM concentration images were nonlinearly warped using diffeomorphic anatomical registration through the exponentiated lie algebra (DARTEL) technique $^{13}$ and were resampled to $2-\mathrm{mm}$ cubic voxels. The GMV of each voxel was obtained by multiplying the GM concentration map by the non-linear determinants derived from the spatial normalization step ${ }^{14}$. Finally, GMV maps were smoothed with a Gaussian kernel with a $6 \mathrm{~mm} \times 6 \mathrm{~mm} \times 6 \mathrm{~mm}$ full-width at half maximum (FWHM). Two researchers performed the analyses independently. Then we check the consistency of results between the two researchers.

CBF calculation. The ASL difference images were calculated after the subtraction of the label images from the control images. The CBF maps were derived from the ASL difference images. The detailed calculation procedures have been described in a previous study ${ }^{15}$. SPM8 software was used to coregister the CBF images of the 95 healthy comparison subjects to a PET-perfusion template in the MNI space using non-linear transformation. The CBF template was defined as the mean CBF image of the 95 healthy comparison subjects. The CBF images of all participants, including patients and controls, were subsequently coregistered to the MNI-standard CBF template and were resampled to $2-\mathrm{mm}$ cubic voxels. The CBF of each voxel was normalized by dividing the mean $\mathrm{CBF}$ of the whole brain ${ }^{16,17}$. Then the resulting CBF maps were spatially smoothed with a Gaussian kernel of $6 \mathrm{~mm} \times 6 \mathrm{~mm} \times 6 \mathrm{~mm}$ FWHM. Two researchers performed the analyses independently. Then we check the consistency of results between the two researchers.

Voxel-based group comparisons. The coregistered GMV and CBF maps with a voxel size of $2 \mathrm{~mm} \times 2 \mathrm{~mm} \times 2 \mathrm{~mm}$ were used for voxel-based group comparisons, which were performed using a two-sample $\mathrm{t}$-test, controlling for the effects of age and sex. Multiple comparisons were corrected using a family-wise error (FWE) method ( $\mathrm{p}<0.05$, two-tailed, cluster size $>100$ voxels). Several steps were used to identify different types of grey matter changes in schizophrenia. First, GMV comparisons between groups were used to differentiate GM regions with and without GMV changes in schizophrenia. Second, group comparisons in CBF were performed in brain regions with GMV reduction in schizophrenia to identify the functional changes in these structurally impaired regions. Third, group comparisons in CBF were performed in brain regions without GMV changes in schizophrenia to identify the functional changes in these structurally normal regions.

Decreased GMV was found in several brain regions in our study, to investigate the potential effect of GMV on CBF, we performed an additional voxel-based group analysis. A general linear model with GMV as an additional covariate was used to recalculate $\mathrm{CBF}$ differences in brain regions with $\mathrm{CBF}$ changes $(\mathrm{p}<0.05$, Bonforroni corrected).

Correlation analyses. To test correlations between different types of brain changes and clinical variables, we drew a sphere with a radius of $6 \mathrm{~mm}$ centred at the centre of gravity of each region, and then we extracted imaging 


\begin{tabular}{|c|c|c|c|c|c|c|c|c|}
\hline \multirow[b]{2}{*}{ Characteristics } & \multicolumn{3}{|c|}{$\begin{array}{l}\text { Healthy comparison } \\
\text { subjects }(\mathrm{n}=95)\end{array}$} & \multicolumn{3}{|c|}{$\begin{array}{l}\text { Schizophrenia patients } \\
(\mathrm{n}=100)\end{array}$} & \multicolumn{2}{|l|}{ Statistics } \\
\hline & Mean & SD & Range & Mean & SD & Range & $t / \chi^{2}$ & $\mathbf{p}$ \\
\hline Age (years) & 33.3 & 10.4 & $20-57$ & 34.1 & 8.2 & $20-59$ & $\mathrm{t}=-0.532$ & 0.595 \\
\hline Sex & M44 & F51 & - & M58 & F42 & - & $x^{2}=2.666$ & 0.103 \\
\hline $\begin{array}{l}\text { Duration of illness } \\
\text { (months) }\end{array}$ & - & - & - & 126.3 & 97.7 & $0-468$ & - & - \\
\hline \multicolumn{9}{|c|}{ Positive and negative syndrome scale score } \\
\hline Positive subscore & - & - & - & 16.9 & 7.8 & - & - & - \\
\hline Negative subscore & - & - & - & 20.2 & 9.0 & - & - & - \\
\hline General subscore & - & - & - & 71.2 & 22.8 & - & - & - \\
\hline $\begin{array}{l}\text { Current } \\
\text { antipsychotic dosage } \\
\text { (chlorpromazine } \\
\text { equivalents) })^{47}(\mathrm{mg} / \mathrm{d})\end{array}$ & - & - & - & 451.8 & 342.3 & $0-1800$ & - & - \\
\hline
\end{tabular}

Table 1. Demographic and clinical characteristics of participants.

measurements of the sphere and calculated Spearman's correlation coefficients between the imaging measurements of the sphere and the clinical parameters (i.e., PANSS score, duration of illness, and antipsychotic dosage). A value of uncorrected $\mathrm{p}<0.05$ was considered significant.

Resting-state functional connectivity calculation. Abnormal GMV and CBF were found in bilateral anterior cingulate cortex (ACC) in our study. To confirm the relationship between ACC and default mode network as well as salience network, we applied a functional connectivity analysis, we regarded each ACC as a region of interest (ROI) and calculate functional connectivity map of each ACC in healthy control subjects (Please see the detailed method in the method section of Supplement 1).

\section{Results}

Demographic and clinical characteristics of subjects. We finally included 100 schizophrenia patients (58 males; age: $34.1 \pm 8.2$ years) and 95 healthy comparison subjects ( 44 males; age: $33.3 \pm 10.4$ years). The demographic and clinical characteristics of these subjects are summarized in Table 1 . There were no significant group differences in sex $\left(\chi^{2}=2.666, p=0.103\right)$ and age $(t=-0.532, p=0.595)$. To further exclude the effects of sex and age on our results, we considered these two variables as covariates of no interest throughout the GMV and CBF analyses. Ninety-two patients were receiving atypical antipsychotics at the time of the MRI examinations; eight patients had never received any medications.

GMV differences between groups. Compared with healthy comparison subjects, schizophrenia patients showed significantly reduced GMV in the bilateral insular cortex, ACC and thalamus and the left middle temporal gyrus (MTG) ( $<<0.05$, FWE correction) (Fig. S1 in the Supplement 1). No significantly increased GMV was observed in schizophrenia patients.

Brain regions with reduced GMV and CBF in schizophrenia. Within brain regions with significant GMV reduction in schizophrenia, we also found significant CBF reduction in the ACC and the left anterior insular cortex ( $\mathrm{p}<0.05$, FWE correction) (Table 2, Fig. 1A). The GMV $(\mathrm{r}=-0.248, \mathrm{p}=0.013)$ and CBF $(\mathrm{r}=-0.325$, $\mathrm{p}=0.001)$ of the ACC and the GMV of the left anterior insular cortex $(\mathrm{r}=-0.230, \mathrm{p}=0.021)$ were negatively correlated with the duration of illness (Fig. 1B,D,F). The GMV of the ACC was negatively correlated with PANSS negative score $(r=-0.277, \mathrm{p}=0.005)$ and the GMV of the left anterior insular cortex was positively correlated with the PANSS positive score $(\mathrm{r}=0.213, \mathrm{p}=0.034)$ in schizophrenia patients (Fig. 1C,E).

Brain regions with reduced GMV and normal CBF in schizophrenia. Within brain regions with significant GMV reduction in schizophrenia, normal CBF was observed in the right insular cortex, the left MPFC, amygdala, superior temporal gyrus (STG) and MTG, and the bilateral thalami and posterior MCC (pMCC) (Table 2, Fig. 2A). The GMVs of the left pMCC $(r=-0.218, p=0.029)$ and MPFC $(r=-0.199, p=0.047)$ were negatively correlated with the duration of illness. The GMVs of the left amygdala $(r=0.242, p=0.015)$ and STG $(r=0.245, p=0.014)$ were positively correlated with the PANSS positive score in schizophrenia patients (Fig. 2B-E).

Brain regions with normal GMV and abnormal CBF in schizophrenia. In brain regions without significant GMV changes, schizophrenia patients showed significantly reduced CBF in the right ACC and the left lateral prefrontal cortex (LPFC) and significantly increased CBF in the bilateral putamen than healthy comparison subjects (Table 2, Fig. 3A,B). The CBF values of the LPFC $(r=-0.269, \mathrm{p}=0.007)$ and ACC $(r=-0.321$, $\mathrm{p}=0.001)$ were negatively correlated with the duration of illness in schizophrenia patients (Fig. 3C,D).

Potential effect of GMV on CBF. We found that CBF alteration remained significant after GMV correction $(\mathrm{p}<0.05$ with Bonforroni corrected) (Fig. S2 in Supplement 1). Thus, CBF alterations are not likely caused by GMV alterations. 


\begin{tabular}{|c|c|c|c|}
\hline Brain regions* & $\begin{array}{l}\text { MNI } \\
\text { coordinates }(\mathrm{x}, \\
\mathrm{y}, \mathrm{z})\end{array}$ & $\begin{array}{l}\text { Peak t } \\
\text { value }\end{array}$ & $\begin{array}{l}\text { Cluster size } \\
\text { (Voxels)*** }\end{array}$ \\
\hline \multicolumn{4}{|c|}{ GMV and CBF reduction } \\
\hline $\begin{array}{l}\text { Anterior cingulate } \\
\text { cortex }\end{array}$ & $2,36,24$ & -7.23 & 699 \\
\hline $\begin{array}{l}\text { Left anterior insular } \\
\text { cortex }\end{array}$ & $-34,22,2$ & -6.38 & 248 \\
\hline \multicolumn{4}{|c|}{ GMV reduction and normal CBF } \\
\hline Right insular cortex & $33,22,-4$ & -7.56 & 1596 \\
\hline $\begin{array}{l}\text { Left medial } \\
\text { prefrontal cortex }\end{array}$ & $-8,50,14$ & -7.12 & 1118 \\
\hline Left amygdala & $-20,2,-18$ & -6.99 & 420 \\
\hline $\begin{array}{l}\text { Left superior } \\
\text { temporal gyrus }\end{array}$ & $-52,0,-2$ & -6.83 & 508 \\
\hline $\begin{array}{l}\text { Right posterior mid- } \\
\text { cingulate cortex }\end{array}$ & $8,18,36$ & -6.57 & 212 \\
\hline $\begin{array}{l}\text { Left posterior mid- } \\
\text { cingulate cortex }\end{array}$ & $-8,22,34$ & -6.20 & 138 \\
\hline $\begin{array}{l}\text { Left middle temporal } \\
\text { gyrus }\end{array}$ & $-60,-36,2$ & -6.02 & 188 \\
\hline Thalamus & $0,-6,4$ & -5.57 & 203 \\
\hline \multicolumn{4}{|c|}{ Reduced CBF and normal GMV } \\
\hline $\begin{array}{l}\text { Right anterior } \\
\text { cingulate cortex }\end{array}$ & $4,36,24$ & -7.14 & 768 \\
\hline $\begin{array}{l}\text { Left lateral prefrontal } \\
\text { cortex }\end{array}$ & $-30,60,2$ & -6.62 & 418 \\
\hline \multicolumn{4}{|c|}{ Increased CBF and normal GMV } \\
\hline Left putamen & $-30,-10,-2$ & 6.38 & 125 \\
\hline Right putamen & $32,-2,2$ & 6.05 & 236 \\
\hline
\end{tabular}

Table 2. GMV, CBF and CBF/GMV ratio changes in schizophrenia patients. *Multiple comparisons are corrected using a family-wise error method ( $\mathrm{p}<0.05$, cluster size $>100$ voxels). **Voxel size is $2 \times 2 \times 2 \mathrm{~mm}^{3}$.

Potential effect of smoothing kernels on GMV and CBF. In addition, we choose a Gaussian kernel of 4 FWHM, 8 FWHM and 10 FWHM to validate whether brain regions showed significantly abnormal GMV and/or $\mathrm{CBF}$ in our study were influenced by the Gaussian kernel. We extracted the signal of GMV or CBF in each significant brain region and adopted a general linear model to compare group differences with sex and age as covariates. The results are listed in Tables S1 and S2 in Supplement 1. We found that the results were still significant when we used other smoothing kernels.

\section{Discussion}

Based on GMV and CBF changes, we identified three types of grey matter changes in schizophrenia: (1) both GMV and CBF reduction in the ACC and anterior insular cortex; (2) GMV reduction with normal CBF in the insular cortex, MPFC, pMCC, STG, MTG, thalamus and amygdala; and (3) CBF reduction with normal GMV in the ACC and LPFC as well as CBF increase with normal GMV in the putamen.

Both GMV and CBF reduction were found in the anterior insular cortex and ACC in schizophrenia patients, indicating both structural and functional impairments in these regions. The anterior insular cortex is an important node of the salience network (SN), which serves to identify and assess salient events, and to initiate appropriate signals to control human behaviors via switching between central executive network and default mode network $(\mathrm{DMN})^{18}$. The structural and functional impairments in the anterior insular cortex is consistent with previous studies that reporting reduced $\mathrm{GMV}^{19}$ and white matter integrity ${ }^{20}$, and abnormal task-evoked activation $^{21}$ in this region in schizophrenia. The impairment of the anterior insular cortex may be an important cause for the dysfunction of the $\mathrm{SN}^{16,22,23}$, which resulting in aberrant salience attribution and contributing to the formation of schizophrenic core symptoms ${ }^{24,25}$. This is confirmed by our correlation finding between the GMV of the anterior insular cortex and PANSS positive score. The resting-state functional connectivity analysis revealed that the impaired ACC region was associated with both the SN and DMN (Fig. S3 in the Supplement 1), suggesting that it is the connected hub of the two networks. Thus, the impairment of the ACC region destructs not only these two networks themselves but also their connections. The DMN plays a role in social cognition and emotional regulation ${ }^{26,27}$, dysfunction of which is frequently reported in schizophrenia ${ }^{28,29}$. The structural and functional impairments in the ACC have been found in both schizophrenia patients and their first-degree relatives ${ }^{4,30}$, ${ }^{31}$. In consistent with previous studies ${ }^{32}$, we found that the structural impairment of the ACC was correlated with clinical symptoms. Consequently, the ACC and the anterior insular cortex, the critical nodes of the SN and DMN, are the most impaired regions in schizophrenia, which may underlie the core dysfunctions in schizophrenia ${ }^{33}$. The structural and functional features of these regions may be used as objective biomarkers for schizophrenia.

Several brain regions with reduced GMV showed normal CBF in schizophrenia. The MPFC and amygdala are components of the corticolimbic circuit which is responsible for affective arousal and regulation. In consistent 

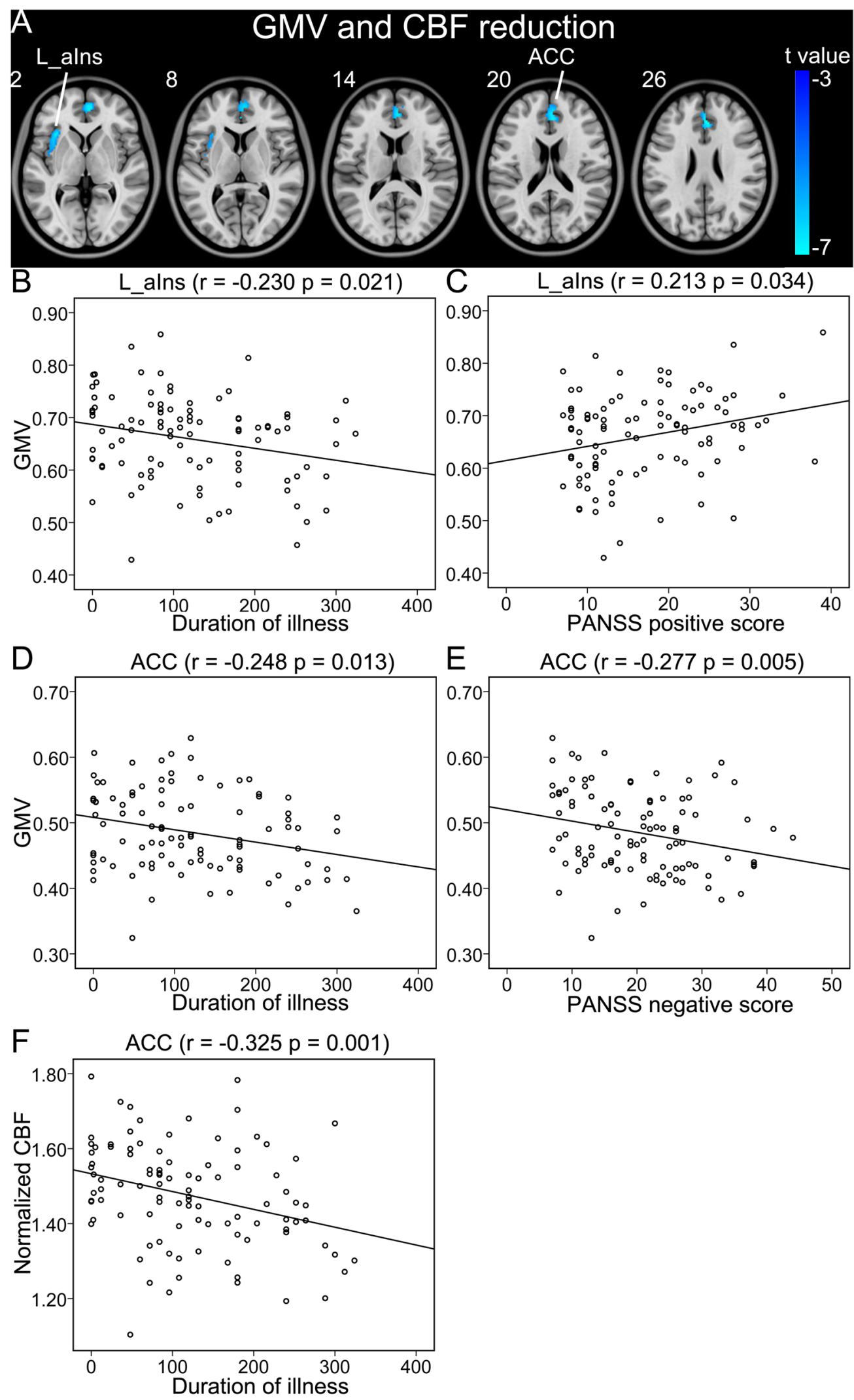

Figure 1. Brain regions with both reduced GMV and CBF in schizophrenia patients (A). (B-F) Shows correlations between GMV and CBF of these regions and clinical parameters in schizophrenia patients. Abbreviations: CBF, cerebral blood flow; GMV, grey matter volume; L, left; and R, right; ACC, anterior cingulate-cortex; aIns, anterior insular cortex. The $r$ value represents the Spearman's correlation coefficients. 

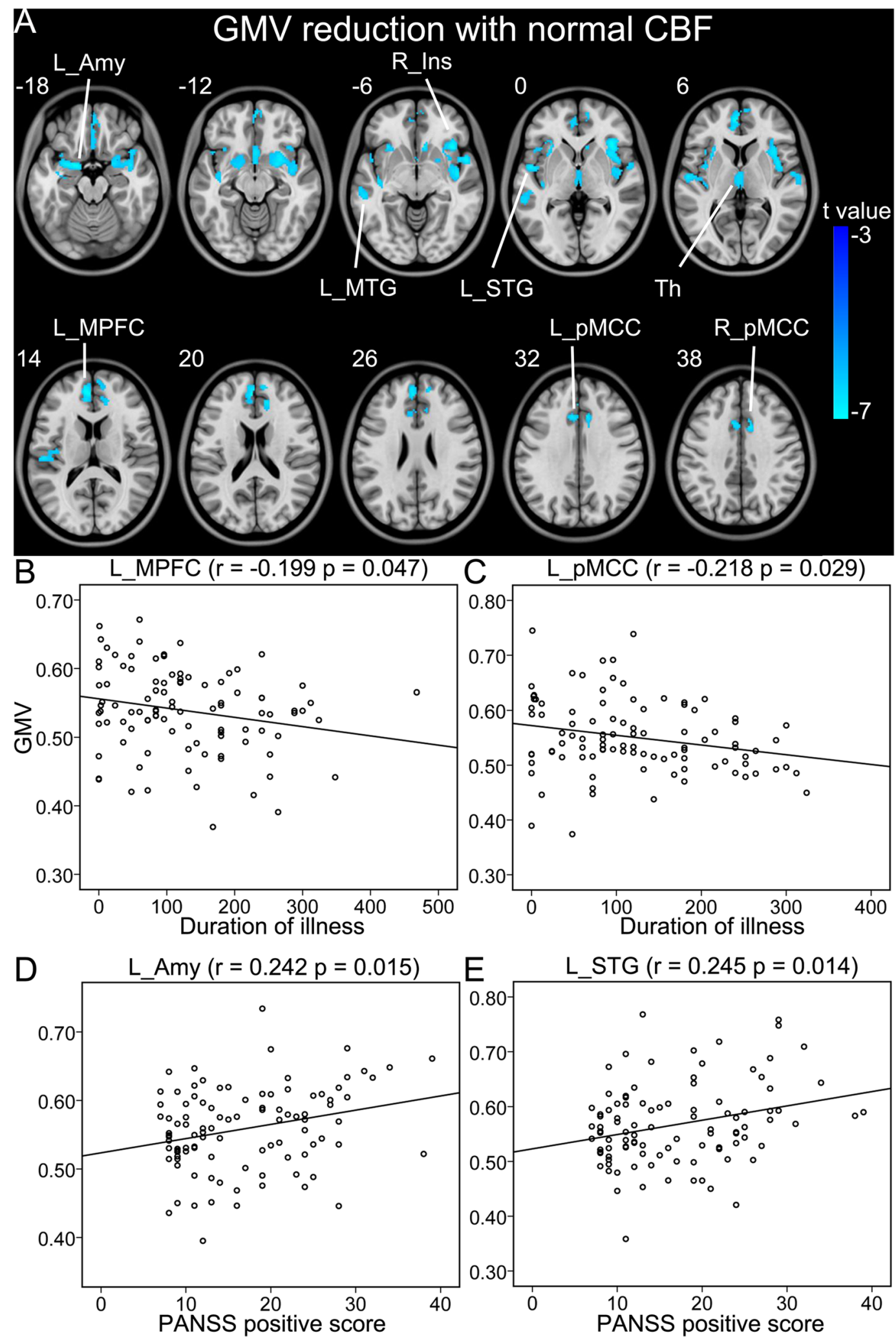

Figure 2. Brain regions with reduced GMV and normal CBF in schizophrenia patients (A). (B-E) Shows correlations between GMV and CBF of these regions and clinical parameters in schizophrenia patients. Abbreviations: CBF, cerebral blood flow; GMV, grey matter volume; L, left; and R, right; Amy, amygdala; Ins, insular cortex; MPFC, medial prefrontal cortex; MTG, middle temporal gyrus; pMCC, posterior middle cingulate-cortex; STG, superior temporal gyrus. The r value represents the Spearman's correlation coefficients. 


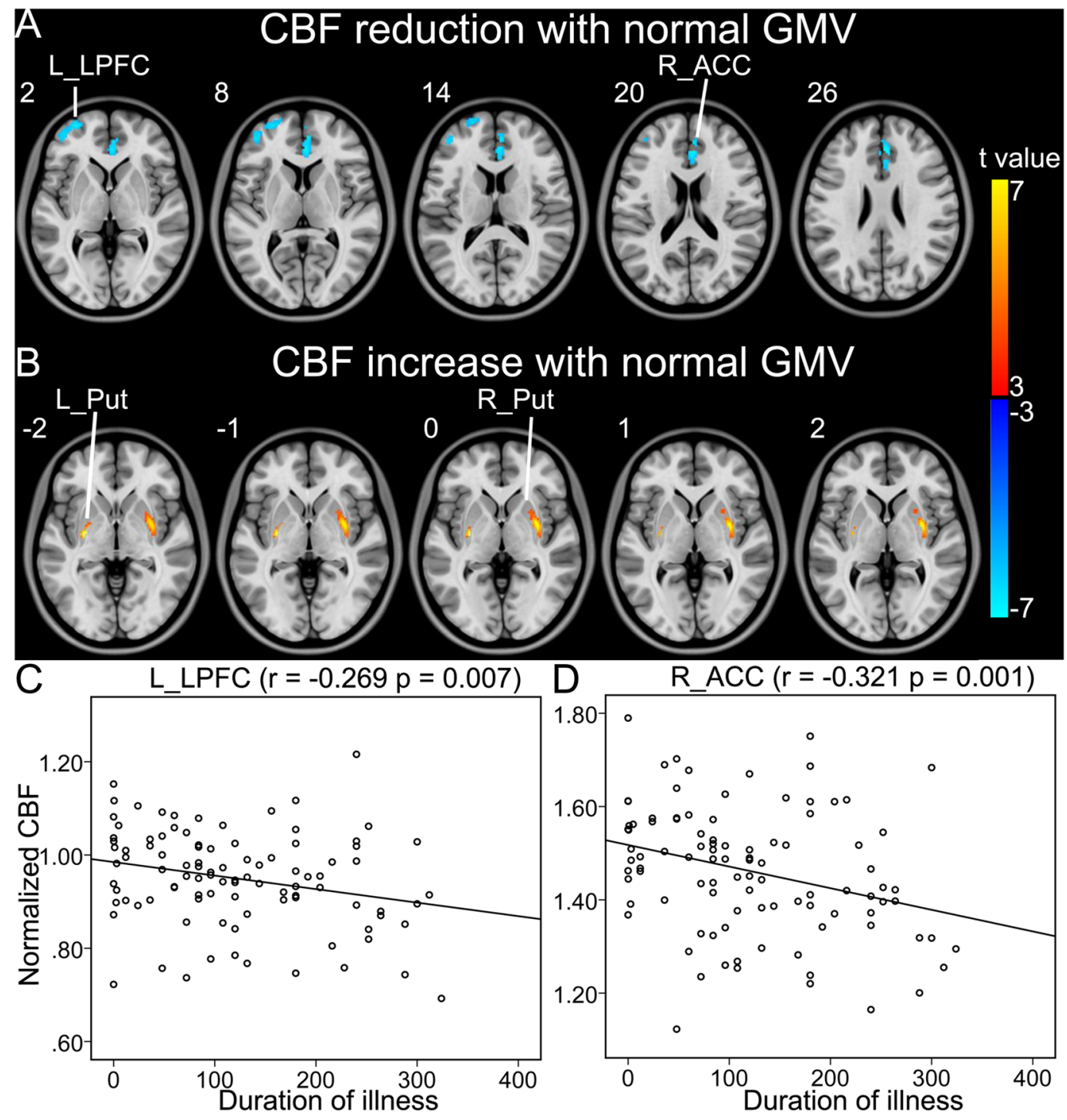

Figure 3. Brain regions with normal GMV and abnormal CBF in schizophrenia patients. (A) Shows brain regions with normal GMV and reduced CBF in patients. (B) Shows brain regions with normal GMV and increased CBF in patients. (C,D) Show correlations between CBF of these regions and clinical parameters in schizophrenia patients. Abbreviations: CBF, cerebral blood flow; GMV, grey matter volume; L, left; and R, right; ACC, anterior cingulate-cortex; LPFC, lateral prefrontal cortex; Put, putamen. The r value represents the Spearman's correlation coefficients.

with a previous study ${ }^{34}$, we found GMV reduction in the MPFC and amygdada. Moreover, we found a positive correlation between the GMV of the amygdala and PANSS positive score, supporting the impairment of this circuit contributing to the affective symptoms in schizophrenia ${ }^{35}$. The pMCC involves in sensorimotor integration and shows functional disconnection in schizophrenia ${ }^{36,37}$, the GMV reduction in the pMCC provides evidence for the structural impairment of this region in schizophrenia. GMV reduction was also found in the thalamus, which has been related to cognitive deficits in schizophrenia ${ }^{38}$. In agreement with previous studies ${ }^{5,39}$, we found GMV reduction in the MTG and STG. These regions involve in auditory and language processing, the impairments of which are associated with core symptoms of schizophrenia, such as auditory hallucination ${ }^{40}$. This finding is further supported by the correlation between the GMV of the left STG and PANSS positive score in our study. The normal CBF in these structurally impaired regions provides evidence of their asynchronization between function and structure impairment, suggesting that effective treatments may prevent these regions from progressing into the stage of functional decompensation.

Among brain regions with normal GMV, several regions displayed abnormal CBF in schizophrenia. As nodes of the SN, the LPFC and ACC showed reduced CBF in schizophrenia, which may also contribute to the dysfunction of the SN. We also found increased CBF in the putamen in schizophrenia, which is consistent with previous perfusion studies ${ }^{9,41}$. The putamen contains rich dopaminergic neurons and is involved in motor, cognition and emotional processing and abnormal activation of putamen was associated with hallucination in schizophrenia ${ }^{42}$. 
The hyper-perfusion in the caudate nucleus has been attributed to antipsychotic treatment ${ }^{43}$; however, the lack of correlation between the CBF of the putamen and antipsychotic dosage in our study and previous studies ${ }^{41,44}$ suggests that whether the striatum is a treatment biomarker needs further validation. The functional abnormalities in structurally normal brain regions may be important targets for antipsychotic treatments, which have the potential to recover these abnormal functions.

In consistent with previous studies reporting progressively structural and functional impairments in schizophrenia ${ }^{45}$, we also found significant negative correlations between the regional GMV and CBF and the duration of illness in schizophrenia patients. These findings suggest that structural and functional integrity may be progressively damaged following disease course of schizophrenia. Therefore, earlier intervention is very important for delaying or preventing the structural and functional impairment in schizophrenia.

There are several limitations that should be mentioned in this study. First, most patients were at the chronic stage of schizophrenia, which prevents us from elucidating the types of brain changes at onset of the disease. This information may help to differentiate brain changes resulted from the developmental or the neurodegenerative mechanism. Second, ninety-two out of 100 schizophrenia patients received antipsychotic treatment during MR scans, we couldn't rule out the possible effect of medication on our findings because increased CBF has been reported in the putamen after the administration of antipsychotic medication ${ }^{46}$. Although we didn't find any significant correlations between imaging parameters and antipsychotic dosage, future studies with medication-naïve, first-episode schizophrenia patients are required to eliminate the possible medication effects in this study. Third, the correlations between imaging measurements and PANSS score presented in our study were not soundly significant, which made our interpretation less convictive.

In summary, we combined the GMV and CBF to identify brain change patterns in schizophrenia. We identified three types of brain changes: structural and functional impairments; structural impairment with preserved function; and pure functional abnormalities. These findings may improve our understanding of the neural mechanisms and facilitate the development of reliable biomarkers for schizophrenia.

\section{References}

1. Haijma, S. V. et al. Brain volumes in schizophrenia: a meta-analysis in over 18000 subjects. Schizophr Bull 39, 1129-1138, doi:10.1093/schbul/sbs118 (2013).

2. Owens, S. F. et al. Prefrontal deviations in function but not volume are putative endophenotypes for schizophrenia. Brain 135, 2231-2244, doi:10.1093/brain/aws138 (2012).

3. Tang, J. et al. Decrease in temporal gyrus gray matter volume in first-episode, early onset schizophrenia: an MRI study. PLoS One 7, e40247, doi:10.1371/journal.pone.0040247 (2012).

4. Salgado-Pineda, P. et al. Correlated structural and functional brain abnormalities in the default mode network in schizophrenia patients. Schizophr Res 125, 101-109, doi:10.1016/j.schres.2010.10.027 (2011).

5. Zhang, W. et al. Brain Structural Abnormalities in a Group of Never-Medicated Patients With Long-Term Schizophrenia. Am J Psychiatry 172, 995-1003, doi:10.1176/appi.ajp.2015.14091108 (2015).

6. Kong, L. et al. Comparison of grey matter volume and thickness for analysing cortical changes in chronic schizophrenia: a matter of surface area, grey/white matter intensity contrast, and curvature. Psychiatry Res 231, 176-183, doi:10.1016/j.pscychresns.2014.12.004 (2015).

7. Harms, M. P. et al. Structural abnormalities in gyri of the prefrontal cortex in individuals with schizophrenia and their unaffected siblings. Br J Psychiatry 196, 150-157, doi:10.1192/bjp.bp.109.067314 (2010).

8. Cha, Y. H. et al. Regional correlation between resting state FDG PET and pCASL perfusion MRI. J Cereb Blood Flow Metab 33, 1909-1914, doi:10.1038/jcbfm.2013.147 (2013).

9. Zhu, J. et al. Altered resting-state cerebral blood flow and its connectivity in schizophrenia. J Psychiatr Res 63, 28-35, doi:10.1016/j. jpsychires.2015.03.002 (2015)

10. Liu, J., Qiu, M., Constable, R. T. \& Wexler, B. E. Does baseline cerebral blood flow affect task-related blood oxygenation level dependent response in schizophrenia? Schizophr Res 140, 143-148, doi:10.1016/j.schres.2012.06.028 (2012).

11. Cabral, C. et al. Classifying Schizophrenia Using Multimodal Multivariate Pattern Recognition Analysis: Evaluating the Impact of Individual Clinical Profiles on the Neurodiagnostic Performance. Schizophr Bull 42 (Suppl 1), S110-117, doi:10.1093/schbul/sbw053 (2016).

12. Kay, S. R., Fiszbein, A. \& Opler, L. A. The positive and negative syndrome scale (PANSS) for schizophrenia. Schizophr Bull 13, 261-276 (1987)

13. Ashburner, J. A fast diffeomorphic image registration algorithm. Neuroimage 38, 95-113, doi:10.1016/j.neuroimage.2007.07.007 (2007).

14. Liu, F. et al. Classification of different therapeutic responses of major depressive disorder with multivariate pattern analysis method based on structural MR scans. PLoS One 7, e40968, doi:10.1371/journal.pone.0040968 (2012).

15. Xu, G. et al. Reliability and precision of pseudo-continuous arterial spin labeling perfusion MRI on $3.0 \mathrm{~T}$ and comparison with 15O-water PET in elderly subjects at risk for Alzheimer's disease. NMR Biomed 23, 286-293, doi:10.1002/nbm.1462 (2010).

16. Wynn, J. K. et al. Impaired target detection in schizophrenia and the ventral attentional network: Findings from a joint event-related potential-functional MRI analysis. Neuroimage Clin 9, 95-102, doi:10.1016/j.nicl.2015.07.004 (2015).

17. Liu, F. et al. Altered Cerebral Blood Flow Covariance Network in Schizophrenia. Front Neurosci 10, 308, doi:10.3389/ fnins.2016.00308 (2016).

18. Seeley, W. W. et al. Dissociable intrinsic connectivity networks for salience processing and executive control. J Neurosci 27, 2349-2356, doi:10.1523/JNEUROSCI.5587-06.2007 (2007).

19. Takahashi, T. et al. Follow-up MRI study of the insular cortex in first-episode psychosis and chronic schizophrenia. Schizophr Res 108, 49-56, doi:10.1016/j.schres.2008.12.029 (2009)

20. Ellison-Wright, I. \& Bullmore, E. Meta-analysis of diffusion tensor imaging studies in schizophrenia. Schizophr Res 108, 3-10, doi:10.1016/j.schres.2008.11.021 (2009).

21. Quintana, J. et al. Brain dysfunctions during facial discrimination in schizophrenia: selective association to affect decoding. Psychiatry Res 191, 44-50, doi:10.1016/j.pscychresns.2010.09.005 (2011).

22. Manoliu, A. et al. Aberrant dependence of default mode/central executive network interactions on anterior insular salience network activity in schizophrenia. Schizophr Bull 40, 428-437, doi:10.1093/schbul/sbt037 (2014).

23. Brookes, M. J. et al. Complexity measures in magnetoencephalography: measuring "disorder" in schizophrenia. PLoS One 10, e0120991, doi:10.1371/journal.pone.0120991 (2015).

24. Palaniyappan, L. \& Liddle, P. F. Does the salience network play a cardinal role in psychosis? An emerging hypothesis of insular dysfunction. J Psychiatry Neurosci 37, 17-27, doi:10.1503/jpn.100176 (2012). 
25. Gaebler, A. J. et al. Auditory mismatch impairments are characterized by core neural dysfunctions in schizophrenia. Brain 138, 1410-1423, doi:10.1093/brain/awv049 (2015).

26. Li, W., Mai, X. \& Liu, C. The default mode network and social understanding of others: what do brain connectivity studies tell us. Front Hum Neurosci 8, 74, doi:10.3389/fnhum.2014.00074 (2014).

27. Ridderinkhof, K. R., Ullsperger, M., Crone, E. A. \& Nieuwenhuis, S. The role of the medial frontal cortex in cognitive control. Science 306, 443-447, doi:10.1126/science.1100301 (2004).

28. Bora, E. \& Pantelis, C. Theory of mind impairments in first-episode psychosis, individuals at ultra-high risk for psychosis and in first-degree relatives of schizophrenia: systematic review and meta-analysis. Schizophr Res 144, 31-36, doi:10.1016/j. schres.2012.12.013 (2013)

29. Morris, R. W. et al. Corticostriatal control of goal-directed action is impaired in schizophrenia. Biol Psychiatry 77, 187-195, doi:10.1016/j.biopsych.2014.06.005 (2015).

30. Meda, S. A. et al. Multivariate analysis reveals genetic associations of the resting default mode network in psychotic bipolar disorder and schizophrenia. Proc Natl Acad Sci USA 111, E2066-2075, doi:10.1073/pnas.1313093111 (2014).

31. Theberge, J. et al. Glutamate and glutamine measured with $4.0 \mathrm{~T}$ proton MRS in never-treated patients with schizophrenia and healthy volunteers. Am J Psychiatry 159, 1944-1946, doi:10.1176/appi.ajp.159.11.1944 (2002).

32. Jessen, F. et al. N-acetylaspartylglutamate (NAAG) and N-acetylaspartate (NAA) in patients with schizophrenia. Schizophr Bull 39, 197-205, doi:10.1093/schbul/sbr127 (2013).

33. Looijestijn, J. et al. An integrated network model of psychotic symptoms. Neurosci Biobehav Rev 59, 238-250, doi:10.1016/j. neubiorev.2015.09.016 (2015).

34. Suzuki, M. et al. Differential contributions of prefrontal and temporolimbic pathology to mechanisms of psychosis. Brain 128, 2109-2122, doi:10.1093/brain/awh554 (2005)

35. Buckholtz, J. W. \& Meyer-Lindenberg, A. Psychopathology and the human connectome: toward a transdiagnostic model of risk for mental illness. Neuron 74, 990-1004, doi:10.1016/j.neuron.2012.06.002 (2012).

36. Wang, D. et al. Altered functional connectivity of the cingulate subregions in schizophrenia. Transl Psychiatry 5, e575, doi:10.1038/ tp.2015.69 (2015).

37. Xu, L. et al. Selective Functional Disconnection of the Dorsal Subregion of the Temporal Pole in Schizophrenia. Sci Rep 5, 11258, doi:10.1038/srep11258 (2015)

38. Pergola, G. et al. The role of the thalamus in schizophrenia from a neuroimaging perspective. Neurosci Biobehav Rev 54, 57-75, doi:10.1016/j.neubiorev.2015.01.013 (2015)

39. Guo, W. et al. Decreased gray matter volume in the left middle temporal gyrus as a candidate biomarker for schizophrenia: a study of drug naive, first-episode schizophrenia patients and unaffected siblings. Schizophr Res 159, 43-50, doi:10.1016/j. schres.2014.07.051 (2014).

40. Hugdahl, K. Auditory hallucinations: A review of the ERC "VOICE" project. World J Psychiatry 5, 193-209, doi:10.5498/wjp. v5.i2.193 (2015).

41. Pinkham, A. et al. Resting quantitative cerebral blood flow in schizophrenia measured by pulsed arterial spin labeling perfusion MRI. Psychiatry Res 194, 64-72, doi:10.1016/j.pscychresns.2011.06.013 (2011).

42. Cui, L. B. et al. Disturbed Brain Activity in Resting-State Networks of Patients with First-Episode Schizophrenia with Auditory Verbal Hallucinations: A Cross-sectional Functional MR Imaging Study. Radiology 160938, doi:10.1148/radiol.2016160938 (2016).

43. Kindler, J. et al. Reduced neuronal activity in language-related regions after transcranial magnetic stimulation therapy for auditory verbal hallucinations. Biol Psychiatry 73, 518-524, doi:10.1016/j.biopsych.2012.06.019 (2013).

44. Allen, P. et al. Resting Hyperperfusion of the Hippocampus, Midbrain, and Basal Ganglia in People at High Risk for Psychosis. Am J Psychiatry 173, 392-399, doi:10.1176/appi.ajp.2015.15040485 (2016).

45. Salisbury, D. F. et al. Progressive and interrelated functional and structural evidence of post-onset brain reduction in schizophrenia. Arch Gen Psychiatry 64, 521-529, doi:10.1001/archpsyc.64.5.521 (2007).

46. Lahti, A. C. et al. Functional effects of antipsychotic drugs: comparing clozapine with haloperidol. Biol Psychiatry 53, 601-608 (2003).

47. Gardner, D. M. et al. International consensus study of antipsychotic dosing. Am J Psychiatry 167, 686-693, doi:10.1176/appi. ajp.2009.09060802 (2010).

\section{Acknowledgements}

This study was supported by grants from the Natural Science Foundation of China (Nos 81425013, 91332113 and 81271551), and the Tianjin Key Technology R\&D Program (14ZCZDSY00018).

\section{Author Contributions}

C.S.Y. designed the experiment. L.X.X. and W.Q. wrote and revised the main manuscript text. C.J.Z., J.J.Z. and H.G.L. prepared the fMRI data and prepared Figures 1-4. All authors reviewed the manuscript.

\section{Additional Information}

Supplementary information accompanies this paper at doi:10.1038/s41598-017-00352-z

Competing Interests: The authors declare that they have no competing interests.

Publisher's note: Springer Nature remains neutral with regard to jurisdictional claims in published maps and institutional affiliations.

(c) (i) This work is licensed under a Creative Commons Attribution 4.0 International License. The images or other third party material in this article are included in the article's Creative Commons license, unless indicated otherwise in the credit line; if the material is not included under the Creative Commons license, users will need to obtain permission from the license holder to reproduce the material. To view a copy of this license, visit http://creativecommons.org/licenses/by/4.0/

(C) The Author(s) 2017 